ORIGINAL ARTICLE

PRACA ORYGINALNA

\title{
CLINICAL SYNDROMES OF THE THALAMIC STROKE IN THE CLASSICAL VASCULAR TERRITORIES: A PROSPECTIVE HOSPITAL-BASED COHORT STUDY
}

DOI: 10.36740/WLek202003115

\author{
Maria M. Prokopiv' ${ }^{1}$, Olena Y. Fartushna ${ }^{2}$ \\ 10. 0. BOGOMOLETS NATIONAL MEDICAL UNIVERSITY, KYIV, UKRAINE \\ 2UKRAINIAN MILITARY MEDICAL ACADEMY, KYIV, UKRAINE
}

\begin{abstract}
The aim: We aimed to determine, describe, and analyze the clinical and neuroimaging features of vascular syndromes of acute thalamic stroke in the classical vascular territories in a prospective hospital-based cohort study.

Materials and methods: We have prospectively recruited 319 acute stroke patients, admitted to the Neurological Center at an academic hospital (Oleksandrivska Clinical Hospital) in Kyiv, Ukraine. Complex neurological, clinical, laboratory, ultrasound, and neuroimaging examinations were performed to all study patients within 24 hours since the symptoms onset.

Results: MRI/CT-proven thalamic stroke was diagnosed in 34 (10.6\%) out of 319 patients, forming a study group. Twenty-two out of 34 patients (average age $61.9 \pm 10.2$ years) were diagnosed with an acute isolated ischemic thalamic stroke, and 12 patients (average age $59.0 \pm 9.6$ years) were diagnosed with an acute thalamic hemorrhage. Conclusions: Specific neurological features of clinical vascular syndromes of acute thalamic stroke in the classical vascular territories were analyzed, compared, and described.
\end{abstract}

KEYWORDS: thalamus, stroke, thalamic stroke, syndrome, vascular territory

\section{INTRODUCTION}

Notably, stroke is one of the leading causes of mortality and long-term disability worldwide, and the economic costs of treatment and post-stroke care are substantial. Every two seconds, someone in the world will have a stroke. [1-5] According to the World Health Organization, 15 million people suffer stroke worldwide each year. Of these, 5 million die and another 5 million are permanently disabled. [6]. Also, more young people are affected by stroke in low- and middle-income countries. $[5,7]$ The highest incidence of stroke occurred in east Asia, followed by the eastern European region, whereas the lowest rates were in central Latin America. $[7,8]$ As populations age, and low-income and middle-income countries go through the epidemiological transition from infectious to non-communicable diseases as the predominant cause of morbidity, together with concomitant increases in modifiable risk factors, it is expected that the burden of stroke will further increase until effective stroke prevention strategies are more widely implemented. [7] Thalamic stroke is not rare, accounting for $11 \%$ of posterior circulation infarcts. [9]

The thalamus plays a critical role in supporting cognitive and motor functions, managing our sensitivity to temperature, light, pain, and physical touch. It controls the flow of visual, auditory, and motor information, being also involved in different aspects of learning, memory, speech, language understanding, emotions, motivation, attention, and wakefulness, being in charge of our sense of balance and awareness of our arms and legs. Medical doctors invariably investigate neurological and neuropsychological symptoms of thalamic strokes during an objective examination of the patient, being an integral part of establishing a syndromological, topical, and clinical diagnosis. [10, 11] However, currently, there are not enough published prospective hospital-based cohort studies that report and analyze pathophysiological features and patterns of the occurrence of neurological, neuropsychological, and clinical vascular syndromes of the thalamic stroke in the classical vascular territories, and their topical diagnosis using clinical, neurological, and neuroimaging methods in a prospective hospital-based cohort study. [10-12]

\section{THE AIM}

The purpose of this study is to describe and analyze the clinical and neuroimaging features of vascular syndromes of thalamic stroke in the classical vascular territories in a prospective hospital-based cohort study, providing a comprehensive clinical and neuroimaging analysis. 


\section{MATERIALS AND METHODS}

The methods of the study, inclusion, and exclusion criteria have been reported in detail previously. $[11,12]$ In brief, only MRI/CT-proven acute thalamic stroke patients age 18 years or older were included in this prospective, hospital-based, cohort study of acute thalamic stroke patients. Institutional ethics board approval was obtained and written informed consent received from all participants or legally authorized representative. All study participants were admitted to the Neurological Center of Oleksandrivska Clinical Hospital, Kyiv, Ukraine, within the first $24 \mathrm{~h}$ since the first stroke symptoms occur. All stroke patients were reviewed by at least two board-certified neurologists with training in cerebrovascular diseases. Clinical history, 12-lead electrocardiogram, blood testing, carotid ultrasound, head CT and/or brain MRI were obtained for all study participants. Stroke education programs were provided to all study participants $[13,14]$.

\section{STATISTICAL ANALYSIS}

Parametric and non-parametric statistic methods were applied. A two-sided $\mathrm{p}<0.05$ was considered significant for all analyses. All statistical analyses were performed using IBM SPSS Statistics Version 22.

\section{RESULTS}

\section{BASIC CHARACTERISTICS OF THE STUDY POPULATION}

In total, 319 adult patients with an acute MRI/CT-proven stroke were screened. Among these 319 patients, 204 (63.9\%) patients were diagnosed with an acute posterior circulation ischemic stroke, and 115 (36.1\%) patients had an intracerebral hemorrhage. Thalamic stroke was diagnosed in 34 $(10.6 \%)$ out of 319 patients, forming a study group. The breakdown for study group by stroke type was as follows:

- 22 patients ( 12 men, 10 women aged 50 to 84 years; average age $61.9 \pm 10.2$ years) were diagnosed with an acute isolated thalamic stroke;

- 12 patients ( 5 men, 7 women aged 57-75 years; average age $59.0 \pm 9.6$ years) with a proven diagnosis of an acute thalamic hemorrhage.

Lacunar Stroke was diagnosed in seven (31.8\%) out of 22 patients with isolated thalamic stroke (foci diameter $\leq 1.5$ $\mathrm{cm}$ ), being more often determined in the posterolateral adjacent zone (in 5 patients) and less often in the inferolateral classical vascular territory of the thalamus (in 2 patients). Non-lacunar stroke, with a diameter of a foci lesion $>1.5 \mathrm{~cm}$, was detected in $15(68.2 \%)$ patients. It arose as a result of occlusion of the thalamic arteries, branches of the posterior cerebral arteries. Ischemic damage to the thalamus in four $(18.2 \%)$ patients was due to cardioembolism. Eleven (50.8\%) patients were diagnosed with atherothrombotic intracranial subtype of ischemic stroke. Nonlacunar thalamic infarcts were mainly localized in the classical thalamic territories (in 11 patients), less often in the border vascular zones (in four patients).
In nine $(40.9 \%)$ out of 22 patients with isolated thalamic stroke, the lesion was localized in the right thalamus, in $12(54.5 \%)$ - in the left thalamus, and in one $(4.6 \%)$ case a bilateral lesion of the thalamus was detected. In our study, thalamic strokes were more often localized in the classical vascular territories - in the territory of the inferolateral artery $(40.9 \%)$ and in the territory of the paramedian artery (27.3\%), and less often - in the borderline vascular zones: posterolateral (22.7\%) and central (9.1\%).

\section{CLINICAL VASCULAR SYNDROMES OF THALAMIC STROKES IN THE CLASSICAL VASCULAR TERRITORIES}

In this article, we describe and analyze thalamic strokes in the paramedian and inferolateral classical vascular territories, as there were no study patients with thalamic strokes in the anterior and/or posterior vascular territories.

In our study group, thalamic stroke in the paramedian vascular territory (in 6-27.3\% of patients) was accompanied by damage to the posteromedian thalamus, including the nucleus of the medial longitudinal fasciculus, the posterior divisions of the dorsomedial and intralaminar nuclei: central, lateral, and parafasicular. Unilateral thalamic stroke in the territory of the paramedian artery causes neuropsychological disturbances, such as a decreased level of consciousness, vertical gaze paresis, cognitive impairment, and personality changes, known as posteromedian thalamic syndrome. Clinically, decreased level of consciousness was detected in all patients: stunning (in 2 patients) was manifested by a restriction of activity and a slowdown in mental reactions; stupor (in 3 patients) was characterized by a deeper depression of consciousness; one patient with bilateral stroke in the territory of the paramedian artery was diagnosed with a deep coma. So, more severe neurological dysfunction and symptoms occurred with a bilateral stroke in the territory of the paramedian artery. It is believed that decreased level of consciousness is a consequence of damage to the posterior parts of the dorsomedial and intralaminar nuclei, as well as an interruption of their connection with the ascending reticular formation and cerebral cortex. [15] Cognitive impairment was manifested with a memory impairment: in one patient with left-sided thalamic lesion in the territory of the paramedian artery, retrograde amnesia (loss of memory for events preceding a stroke) was revealed, and in two patients with right-sided thalamic stroke, anterograde amnesia (loss of memory for events after a stroke) was determined. In one patient with left hemisphere lesion in the territory of the paramedian artery, ideomotor and constructive apraxia were detected. In another case with a lesion in the territory of the paramedian artery of the right thalamus, anosognosia, hemineglect with ignoring the left side of space, and distorted perceptions of reality in the form of hallucinosis were observed. In right-handed patients with left-thalamus lesions, speech disorders were noted. Vertical paresis of the gaze up was detected in three patients with a lesion in the territory of the paramedian artery of the left thalamus. Thalamic stroke 
in the territory of the paramedian artery manifested by behavioral syndromes - anomia and dysthymia.

Thalamic stroke in the inferolateral classical vascular territory was developed in nine (40.9\%) out of 22 study patients. Different degrees of surface sensitivity impairment of the contralateral half of the body were the main neurological symptoms noted in these patients. Hemigipesthesia in six (66.7\%) patients was combined with batihypesthesia and contralateral hemiataxia. In seven $(77.8 \%)$ patients, stroke lesion spread to the adjacent internal capsule, causing sensorimotor syndrome on the opposite to the lesion side. At the same time, in two patients, motor impairment preceded by the sensitivity impairment (sensorimotor stroke). In other five patients, motor and sensitivity impairment coincided in time. In four patients with left-sided lesions in the territory of the inferolateral artery of the thalamus, varying degrees of the emotional sphere dysfunction and various manifestations of dysthymia (longing, apathy, fear) were noted. The most common causes of thalamic stroke in the territory of the inferolateral artery were microangiopathies in patients with arterial hypertension and hypercholesterolemia (five patients), diabetes mellitus (two patients), cardioembolism (two patients).

\section{DISCUSSION}

There is a relative paucity of data in the English literature that describe and analyze the clinical and neuroimaging features of vascular syndromes of thalamic stroke in the classical vascular territories in a prospective hospital-based cohort study of Eastern European patients. Based on the vascular supply, neuroanatomical, and neuropathological data, confirmed by neuroimaging techniques, thalamic infarcts have been classically categorized into four thalamic territories, and they are as follows:

1.anterior territory (supplied by the polar/tuberothalamic arteries that arise from the posterior communicating artery);

2.paramedian (supplied by the paramedian arteries that arise from the P1 segment of the posterior cerebral artery);

3.inferolateral (supplied by the thalamogenicular arteries that arise from the P2 segment of the posterior cerebral artery);

4.posterior (supplied by the posterior choroidal arteries that arise from the P2 segment of the posterior cerebral artery).

Our fundings indicates that unilateral stroke in the territory of the paramedian artery manifested with the posteromedian thalamic syndrome. Impaired consciousness and memory, paresis of the gaze up, pronounced neuropsychological disorders, and hallucinosis were the main symptoms of this syndrome. It is believed that the damage to the posterior parts of the dorsomedial and intralaminar nuclei, as well as an interruption of their connection with the ascending reticular formation and the cerebral cortex, causes decreased level of consciousness. [16] Stroke in the territory of the paramedian artery generates excessive im- pulse to the temporal lobe cortex, which is involved in the perception and processing of visual information, causing the development of hallucinosis. [16] The occurrence of selective upward disturbance of the gaze confirms that thalamic stroke in the territory of the paramedian artery has pathological effect on the supranuclear tracts responsible for vertical control of the gaze without damage to the rostral midbrain. [17]

We found that more severe neurological dysfunction occurred in patients with bilateral stroke in the territory of the paramedian artery. Paramedian thalamic stroke syndrome with akinetic mutism, amnestic disorders, occurred when the dorsomedial nuclei of the thalamus were affected. In our observation, a thalamic dementia was developed within a year after a stroke in a patient with bilateral stroke in the territory of the paramedian artery. Thalamic dementia occurs when the medial dorsal nuclei of the thalamus were damaged along with the mamillary bodies. [18] Bilateral stroke in the territory of the paramedian artery occurs due to atheromatous or embolic occlusion of the common branch of the thalamosubtalamic artery known as Percheron's artery.

Isolated thalamic stroke in the territory of the inferolateral artery manifested by heterolateral hemihypesthesia and contralateral hemiataxia. Sensitivity impairments were often combined with impaired motor skills due to a damage to the tissue of the internal capsule adjacent to the stroke foci, determining the development of the sensorimotor syndrome.

In patients with extensive inferolateral thalamic stroke, the classic thalamic Dejerine-Roussy syndrome occurred. This, so-called thalamic pain syndrome, characterized by moderate contralateral hemiparesis, hemihypesthesia, hemiataxia, hemialgia, paresthesia, as well as dysesthesia (perversion of the perception of sensitive irritations). Dejerine-Roussy syndrome is a neurologic disorder first described by the French neurologist, Jules Joseph Degerin and his student Gustave Roussy, in 1906. Thalamic pain syndrome was revealed in two patients with an extensive ischemic stroke in the territory of the inferolateral artery, being characterized by the following clinical manifestations: moderate transient hemiparesis on the opposite lesion side without side signs of muscle spasticity, severe hemihypesthesia, hemiataxia, pain and paresthesia after a stroke in the distal extremities, often extending to the entire half of the body - hemialgii.

In patients with extensive lesions in the inferolateral vascular territory of the thalamus, a dynamic contracture of the fingers occurred in the opposite to the stroke side, so-called, classic thalamic arm "main thalamic" that is described as follows: the forearm is bent and penetrated, the wrist joint is bent, the main phalanges of the fingers are moderately bent, while the middle and end phalanges are fully extended. The fingers of the hand are in continuous motion - choreoathetosis. [11] The described clinical syndrome occurs as a result of damage to the posterior ventral nucleus and ventrolateral thalamic nucleus with the spread of the infarction foci to the inner capsule. 
Neurological symptoms in patients with thalamic stroke in the territory of the inferolateral artery can mimic symptoms of ischemic stroke with capsular localization of the lesion (carotid arterial systems). Thalamic pain with all signs of hyperpathia, emotional and autonomic manifestations was pathognomonic for the thalamic stroke. Thalamic syndrome was associated with hemianesthesia, hemiataxia, and hemianopsia even though these symptoms are not pathognomonic for thalamic stroke. In addition to the sensitivity impairment, hemialgia, paresthesia, psychosensory impairment (hallucinosis), choreoathetosis, dystonic manifestations with a tendency to contractures of a dynamic type, and emotional central paresis of facial muscles were noticed. In all patients with thalamic stroke in the territory of the inferolateral artery, varying degrees of cognitive status impairments were present. The most significant cognitive impairment was observed in patients with Degerin-Roussy syndrome.

\section{CONCLUSIONS}

Summarizing, we would like to highlight that the unilateral thalamic stroke in the territory of the paramedian artery typically manifested by the posteromedian syndrome (decreased level of consciousness, paresis of the gaze up, cognitive impairment); more severe symptoms were observed in a case of a bilateral stroke (paramedian thalamic infarction syndrome). Severe emotional dysfunction, such as syndromes of anomie and dysthymia, were typical in patients with thalamic stroke in the territory of the paramedian artery.

Isolated thalamic stroke in the territory of the inferolateral artery manifested by a syndrome of heterolateral hemianesthesia, hemataxia, combined with impaired motor skills, the presence of a pain, autonomic disorders with signs of hyperpathia, cognitive deficit; a specific sign was the development of thalamic syndrome Dejerine-Roussy; with an extensive stroke lesions, a dynamic contracture of the fingers of the hand occurred - the classic "thalamic arm" ("main thalamic"); the spread of a stroke to the adjacent inner capsule caused the development of sensorimotor syndrome. The understanding of the vascular syndromes of the thalamic strokes was and remains an extremely important component of the supervision of a patient with thalamic stroke, and is an integral part of establishing a topical and clinical diagnosis.

\section{REFERENCES}

1. World Stroke Organization. Global Stroke Fact Sheet. 26.02.2019. Available from: https://www.world-stroke.org/images/WSO_Global_ Stroke_Fact_Sheet_final.pdf

2. Vinychuk S, Fartushna OYe. Cerebrospinal and commissural diaschisis in acute stroke patients: case analysis. International Neurological Journal. 2018;5(99):20-25. https://doi.org/10.22141/22240713.5.99.2018.142959

3. Fartushna OYe, Vinychuk S. Brain injury in patients with acute TIA: clinical features in different TIA subtypes. International Neurological Journal. 2017;3(89):13-18. https://doi.org/10.22141/22240713.3.89.2017.104238
4. VinychukS.M, Prokopiv M.M. Gostryj ishemichnyj insul't [Acute ischemic stroke]. Kyiv: Naukova dumka; 2006, p. 286 (in Ukrainian) ISBN: 96600-0615-2

5. Fartushna OYe, Vinychuk SM. Tranzytorni ishemichni ataky [Transient Ischemic Attacks]. Kyiv: PH «Avitsena»; 2014, p. 216 (in Ukrainian). ISBN 978-966-2144-70-3

6. Institute for Health Metrics and Evaluation (IHME). Findings from the Global Burden of Disease Study 2017. Seattle, WA: IHME, 2018.

7. GBD 2016 Causes of Death Collaborators. Global, regional, and national age-sex specific mortality for 264 causes of death, 1980-2016: a systematic analysis for the Global Burden of Disease Study 2016. Lancet. 2017;390(10100):1151-1210. http://doi.org/10.1016/S01406736(17)32152-9

8. Fartushna 0.Ye, VinychukS.M. Epidemiology of transient ischemic attacks in the structure of acute cerebrovascular disorders in Ukraine and in other countries. International Neurological Journal. 2017;5(91):105-111. (in Ukrainian). http://doi.org/10.22141/2224-0713.5.91.2017.110863

9. Chen $X Y$, Wang $Q$, Wang $X$, et al. Clinical Features of Thalamic Stroke. Curr Treat Options Neurol. 2017;19(2)-5. http://doi.org/10.1007/s11940017-0441-x.

10. Cheng H, Tian Y, Hu P., Wang J, \& Wang, K. Time-based prospective memory impairment in patients with thalamic stroke. Behavioral Neuroscience, 2010;124(1), 152-158. D0l: http://dx.doi.org/10.1037/ a0018306

11. VinychukS.M, Prokopiv M.M, Trepet L.N. Talamičeskie insul'ty [Thalamic stroke]; 0.0.Bogomolets Medical University, Oleksandrivska Clinical Hospital, Kyiv, Ukraine.Kyiv: Agata-Print, 2018; p. 91 (in Russian)

12. Vinychuk S.M, Prokopiv M.M, Trepet L.M, Fartushna OY. Thalamic stroke outcomes: a prospective hospital-based cohort study. International Neurological Journal. 2019;8(110):23-27. http://doi. org/10.22141/2224-0713.8.110.2019.187888

13. Adams R, Albers $G$, Albers $M$, et al. Update to the AHA/ASA Recommendations for the Prevention of Stroke in Patients With Stroke and Transient Ischemic Attack. Stroke. 2008;39:1647-1652. https://doi. org/10.1161/STROKEAHA.107.189063

14. Vinychuk SM, Fartushna OYe. Early rehabilitation after acute ischemic cerebrovascular events. International Neurological Journal. 2016;8(86):34-39. (in Ukrainian) http://dx.doi.org/10.22141/22240713.8.86.2016.90909

15. Bassetti C, Mathis J, Gugger M et al. Hypersomnia following paramedian thalamic stroke: a report of 12 patients. Ann Neurol. 1996;39(4):471480. http://doi.org/10.1002/ana.410390409

16. Middleton F, Strick P. The temporal lobe is a target of output from the basal ganglia. Proc. Natl. Acad. Sci. USA, 1996;93(16):8683-8687. http:// doi.org/10.1073/pnas.93.16.8683

17. Clark J.M., Alberts G.W. Vertical gaze palsies from medial thalamic infarctions without midbrain involvement. Stroke.1995; 26(8):14671470. http://doi.org/10.1161/01.str.26.8.1467

18. Victor M, Adams R, Collins G. The Wernicke-Korsakoff syndrome: a clinical and pathological study of 245 patients, 82 with post-mortem examinations. Contemp Neurol Ser. 1971; 7: 1-206. PMID: 5162155

This article is the part of the research topic named "To determine the features of the course and consequences of a stroke in patients of different age groups, taking into account genetic, infection factors, and comorbid pathologies" for 2018-2020 with the state registration number - $0118 U 003695$ 
ORCID and contributionship:

Maria M. Prokopiv - 0000-0001-5467-3946 A, B, C, E, F

Olena Y. Fartushna - 0000-0002-4641-0836 A,C,D,E,F

\section{Conflict of interest:}

The Authors declare no conflict of interest.

\section{CORRESPONDING AUTHOR}

\section{Olena Y. Fartushna}

Department of Aviation Marine Medicine and Psychophysiology,

Ukrainian Military Medical Academy

Melnikova St., 24, Kyiv, 04050, Ukraine;

e-mail: olena.y.fartushna@gmail.com

tel: +380662683527

Received: 24.01 .2020

Accepted: 05.03.2020

A - Work concept and design, B - Data collection and analysis, C - Responsibility for statistical analysis,

$\mathbf{D}$-Writing the article, $\mathbf{E}$-Critical review, $\mathbf{F}$ - Final approval of the article 